\title{
Low dose irradiation increases adoptive cytotoxic T lymphocyte migration in gastric cancer
}

\author{
JUAN DU, SHU SU, HONGYAN LI, JIE SHAO, FANYAN MENG, MI YANG, HANQING QIAN, \\ ZHENGYUN ZOU, XIAOPING QIAN and BAORUI LIU
}

\begin{abstract}
The Comprehensive Cancer Center of Drum Tower Hospital, The Affiliated Drum Tower Clinical Medical College of Nanjing Medical University, Nanjing, Jiangsu 210008, P.R. China
\end{abstract}

Received January 22, 2016; Accepted February 3, 2017

DOI: $10.3892 /$ etm.2017.5305

\begin{abstract}
Adoptive cellular immunotherapy (ACI) has been demonstrated to be a promising cancer therapeutic; however, the inefficient migration of adoptive immune cells to tumors is one of the rate-limiting factors of ACI. The present study investigated whether 2 Gy low dose irradiation (LDI) was able to increase the migration of adoptive lymphocytes to gastric cancer cells. Treatment with 2 Gy LDI resulted in marked chemokine (C-X-C motif) ligand 9 (CXCL9) and CXCL10 production from gastric cancer cell lines. A Transwell chamber migration assay demonstrated enhanced transmigration of cytotoxic $\mathrm{T}$ lymphocytes to gastric cancer cells following LDI treatment. After 2 Gy LDI application to established gastric carcinoma in nude mice, labeled immune cells were infused by intravenous injection and concentrated fluorescence signals were observed at the tumor sites within the mice, with a peak signal at 8-h LDI. Increased numbers of adoptive $\mathrm{T}$ cells at the tumor sites were also observed using flow cytometry. Furthermore, a case study of a patient with metastatic gastric cancer who had received ACI treatment combined with 2 Gy LDI provided further evidence that 2 Gy LDI is able to recruit antitumor effector $\mathrm{T}$ cells to tumor sites. Therefore, the ability of 2 Gy LDI to convert tumors into inflamed peripheral tissues may be exploited to overcome obstacles at the effector phase of the antitumor immune response and improve the therapeutic efficacy of immunotherapy.
\end{abstract}

\section{Introduction}

Adoptive cellular immunotherapy (ACI), a developing cancer therapeutic, is able to mobilize and strengthen the body's immune system to kill cancer cells; however, clinical trials using ACI

Correspondence to: Professor Baorui Liu, The Comprehensive Cancer Center of Drum Tower Hospital, The Affiliated Drum Tower Clinical Medical College of Nanjing Medical University, 321 Zhongshan Road, Nanjing, Jiangsu 210008, P.R. China E-mail: baoruiliu@nju.edu.cn

Key words: gastric carcinoma, adoptive cellular immunotherapy, low-dose irradiation, chemokines, migration have yielded low objective response rates (1). Inefficient T cell migration limits the effect of cancer immunotherapy. Even large numbers of fully activated tumor-specific cluster of differentiation (CD) $4^{+} \mathrm{T}$ helper 1 and cytotoxic $\mathrm{CD}^{+} \mathrm{T}$ cells may fail to reject established immunogenic murine or human tumors due to their insufficient migration to tumor tissue $(2,3)$. An essential requirement for cancer immunotherapy is activation of antigen-specific T cells and their migration to tumor sites (4).

Radiotherapy has a synergistic effect on immunotherapy. Low dose irradiation (LDI) of 2 Gy had been demonstrated to induce the highest ratio of effector T cells to FoxP3 ${ }^{+}$immunosuppressive regulatory $\mathrm{T}$ cells when combined with adoptive $\mathrm{T}$ cell transfer (5). However, no previous reports have investigated combining 2 Gy LDI with ACI to increase the migration capacity of adoptive lymphocytes to tumor cells. The distribution of immune cells injected into a tumor-bearing body after 2 Gy LDI has not yet been described. Therefore, it would be worthwhile to acquire visual evidence of the migration ability of immune cells after 2 Gy LDI.

Chemokines and their receptors have a crucial role in $\mathrm{T}$ cell recruitment to different tissues, and regulate both homeostatic and inflammation-dependent homing of T cells (6-8). However, little is understood about the chemokines involved in the recruitment of effector $\mathrm{T}$ cells to tumors following irradiation. Cancer cells produce several chemokines, largely to recruit leukocytes that promote tolerance and immune escape, and to aid tumor growth by enhanced angiogenesis. On the other hand, chemokine (C-X-C motif) ligand 9 (CXCL9) and CXCL10 have been demonstrated to recruit lymphocytes that reject the tumors $(9,10)$.

Owing to the progression of fluorescence imaging techniques, it is possible to trace the migration of cells in living animal models (11). The development of a near-infrared fluorescence imaging technique has made it possible to trace living cells in deep tissue (12). Furthermore, fluorescent labeling of immune cells with 1,1'-dioctadecyl-3,3,3',3'-tetramethy indotricarbocyanine iodide (DiR) does not adversely affect their proliferation and cytotoxic function, and the labeling efficiency is $\sim 99.9 \%$ (13).

In the present study, a AGS-EBV preclinical model of metastatic gastric adenocarcinoma was utilized to determine whether 2 Gy LDI is able to increase the migration capacity of cytotoxic T lymphocytes (CTLs) to tumor cells. Whether LDI 
increased the expression and release of chemokines CXCL9 and CXCL10 by AGS-EBV cells and recruitment of tumor-specific activated $\mathrm{CD}^{+}$cells will be discussed. The present study monitored antigen-specific CTLs dynamically labeled with the near-infrared fluorescent dye, DiR. The present study highlights the therapeutic potential of local LDI combined with ACI.

\section{Materials and methods}

Ethics statement. All animal protocols followed the experimental procedures of the National Institutes of Health Guide for the Care and Use of Laboratory Animals and were approved by the Ethics Committee of Drum Tower Hospital. All donors provided their informed written consent.

Cell line. Human gastric adenocarcinoma cell line AGS-EBV (purchased from the Shanghai Institute of Biochemistry and Cell Biology, Chinese Academy of Sciences, Shanghai, China) was cultured in RPMI-1640 media (Sigma-Aldrich; Merck Millipore, Darmstadt, Germany) supplemented with $10 \%$ fetal bovine serum (Invitrogen; Thermo Fischer Scientific, Inc.) and $1 \mathrm{U} / \mathrm{ml}$ gentamicin (Gibco; Thermo Fisher Scientific, Inc.). The culture was maintained in a humidified atmosphere of $5 \% \mathrm{CO}_{2}$ at $37^{\circ} \mathrm{C}$.

Antigen peptides. Epstein-Barr virus (EBV)-latent membrane protein (LMP)2A-derived peptide LMP2A356-364 (FLYALALLL) was synthesized according to standard Fmoc solid phase synthesis methods (GenScript, Nanjing, China). Peptide purity was determined using analytical reverse-phase high performance liquid chromatography by GenScript Corporation (Nanjing, China) and purity was routinely $>95 \%$. Lyophilized peptides were diluted in dimethyl sulfoxide and stored at $-20^{\circ} \mathrm{C}$.

Animals. A total of 30 female BALB/c nu/nu nude mice (6- to 8-weeks old; weight, $20 \pm 2 \mathrm{~g}$ ) were obtained from the Animal Center of the Nanjing University Medical School (Nanjing, China). All animals were breed in a specific pathogen-free class barrier system, had access to a 12-h light/dark cycle and were maintained in a temperature controlled room $\left(20-26^{\circ} \mathrm{C}\right)$ with a humidity of $50-80 \%$. Mice had free access to water and food.

Induction of LMP2A-specific CTLs by LMP2A peptide pulsed myeloid dendritic cells ( $m D C s$ ) in vitro. Human CTLs of healthy donors were generated as described previously (14). In brief, mDCs were harvested after 5 days of culture in the presence of recombinant human (rh) granulocyte macrophage colony-stimulating factor $(200 \mathrm{ng} / \mathrm{ml})$ and rh interleukin (IL)-4 (100 ng/ml; both PeproTech, Inc., Rocky Hill, NJ, USA), adjusted to $5 \times 10^{5}$ cells $/ \mathrm{ml}$ in 24-well plates and incubated at $37^{\circ} \mathrm{C}$ for $48 \mathrm{~h}$ with EBV-LMP2A peptide $(25 \mu \mathrm{g} / \mathrm{ml})$. Tumor necrosis factor (TNF)- $\alpha(200 \mathrm{ng} / \mathrm{ml}$; PeproTech, Inc.) was added to the culture media, which was then incubated at $37^{\circ} \mathrm{C}$ for $24 \mathrm{~h}$. T cells were co-cultured with LMP2A-DCs at a ratio of 10:1 in 24-well plates in $1 \mathrm{ml}$ AIM-V medium (Life Technologies; Thermo Fisher Scientific, Inc.) supplemented with 10\% human AB serum (Gibco; Thermo Fisher Scientific, Inc.) at $37^{\circ} \mathrm{C}$ in $5 \% \mathrm{CO}_{2}$ for 5-7 days. Cells were stimulated with fresh peptide-pulsed autologous DCs twice daily for 7 days. After 3 days, rhIL-2 was added at a final concentration of $20 \mathrm{U} / \mathrm{ml}$ to all wells and this was repeated every 2-3 days thereafter. On day seven after the final stimulation, cells were harvested and LMP2A356-364 epitope-specific cytolytic activity of $\mathrm{T}$ lymphocytes was measured using tetramer technology. Briefly, the single cell suspension was adjusted to $5 \times 10^{6}$ cells $/ \mathrm{ml}$ and $1 \mathrm{ml}$ was inserted into a $1.5-\mathrm{ml}$ Eppendorf tube. Following washing two times with phosphate buffered saline (PBS), the cells were suspended in $5 \mu$ l phycoerythrin-labeled LMP2A-356 tetramer (Kuangbo Biotechnology Corp, Beijing, China), incubated in the dark at $4^{\circ} \mathrm{C}$ for $20 \mathrm{~min}$ and washed two times with PBS again. Subsequently, $2.5 \mu \mathrm{l}$ PerCP-Cy5.5-labeled human CD3 antibodies (BD Biosciences, Franklin Lakes, NJ, USA; cat. no. 6203-25; 1:40 dilution) and $2.5 \mu \mathrm{l}$ allophycocyanin-labeled human CD8 antibodies (BD Biosciences; cat. no. 641400; 1:40 dilution) were added and the mixture was incubated in the dark at $4^{\circ} \mathrm{C}$ for $20 \mathrm{~min}$. Subsequently, cells were washed with PBS two times again and suspended to $300 \mu \mathrm{l}$ PBS prior to being examined using a flow cytometer. Data was analyzed using CellQuest software (Version 5.1, BD Biosciences).

Detection of secretion of chemokines in tumor cells. Gastric carcinoma AGS-EBV cells were incubated in RPMI-1640 medium with no serum for $12 \mathrm{~h}$ to starve the cells. Subsequently, the cells were plated at $1 \times 104$ cells/well in duplicate wells of a 96-well plate. Chemokine concentrations were measured $24 \mathrm{~h}$ after 2 or 5 Gy radiation or mock treatment. CXCL9 and CXCL10 release in culture supernatant was determined using ELISA kits (RayBiotech, Inc., Norcross, GA, USA; cat. nos. QC123 and QC23).

Transwell migration assay. Transwell migration assays were performed using 3- $\mu \mathrm{m}$ Transwell chambers (Corning, Inc., Corning, NY, USA). Briefly, $5 \times 10^{5} \mathrm{~T}$ cells were added to the upper chamber in $100 \mu \mathrm{l}$ of AIM V supplemented with $1 \%$ bovine serum albumin (chemotaxis buffer) (Life Corporation). The lower chamber contained $600 \mu \mathrm{l}$ of chemotaxis buffer and $1 \times 10^{5}$ AGS-EBV cells $24 \mathrm{~h}$ after they had been irradiated with 2 or 5 Gy or not treated. After incubation for $4 \mathrm{~h}$ at $37^{\circ} \mathrm{C}$, cells that migrated inzto the lower chamber were collected and counted.

Fluorescent labeling of immune cells. DiR is a lipophilic, near-infrared fluorescent cyanine dye used for labeling the cytoplasmic membrane. CTLs were suspended at a concentration of $1 \times 10^{6} \mathrm{cells} / \mathrm{ml}$. DiR working solution $(1 \mu \mathrm{g} / \mathrm{ul})$ was added into the cell suspension and incubated for $30 \mathrm{~min}$ at $37^{\circ} \mathrm{C}$ with $10 \mathrm{ul}$ of dye used per $10^{6}$ cells. Following this, the dye was cleared away with two washes using AIM V medium.

Establishment of a gastric carcinoma model. A total of 20 female nude mice were injected subcutaneously with $1 \times 10^{7}$ AGS-EBV cells. After tumor formation (2-3 week later), nude mice bearing gastric carcinomas were randomly divided into two groups: Intravenous injection of CTLs (CTL-i.v.) group and intravenous injection of CTLs plus local $2 \mathrm{~Gy} \mathrm{LDI}$ at tumor sites (CTL-i.v. + LDI) group.

Adoptive transfer and LDI. Tumors were either not irradiated or locally irradiated with a 2-Gy dose of radiation using an X-RAD 


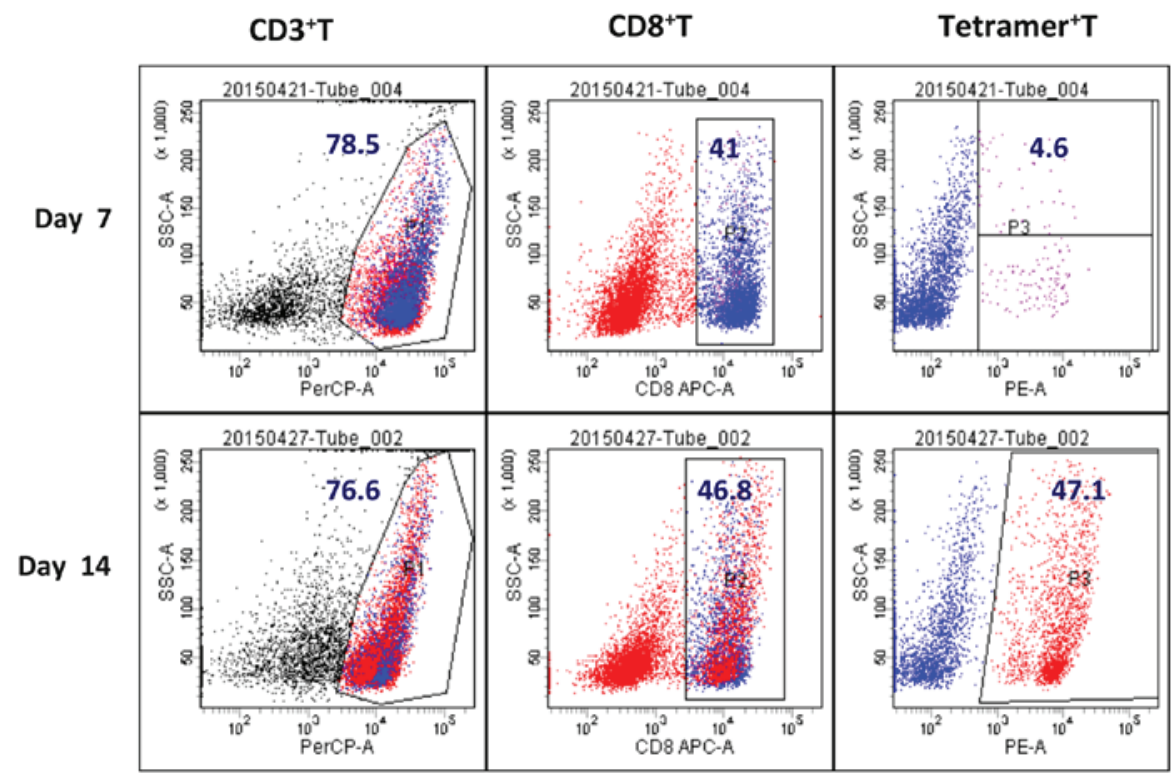

Figure 1. Contents of LMP2A-specific CTLs. EBV-LMP2A-356-specific CTLs accounted for 47.1\% of CD8 ${ }^{+}$T cells 14 days after cell culture. LMP2A, latent membrane protein 2A; EBV, Epstein-Barr virus; CTLs, cytotoxic T lymphocytes; CD, cluster of differentiation.

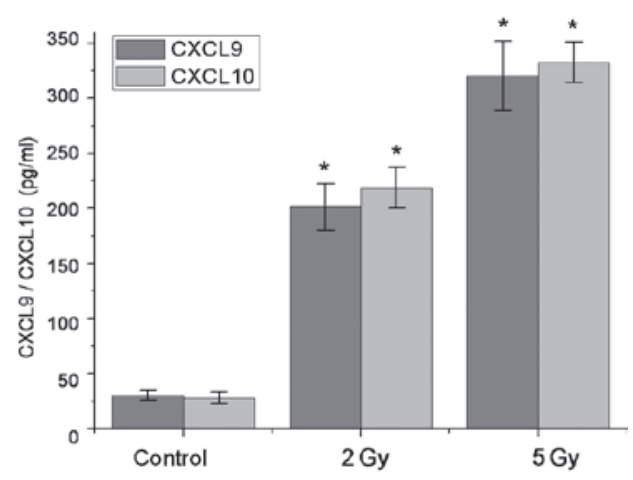

Figure 2. Induction of chemokine CXCL9 and CXCL10 in a human gastric cancer cell line using LDI. Soluble chemokines CXCL9 and CXCL10 in irradiated tumor cells were significantly increased by $\sim 7$ - and 10 -fold in the 2 and 5 Gy groups, respectively, compared with non-irradiated control tumor cells. Data are present as the mean \pm standard deviation. "P $<0.05$ vs. Control. CXCL, (C-X-C motif) ligand; LDI, low dose irradiation.

320 Biological Irradiator (Precision X-Ray, North Branford, CT, USA) 1 day prior to transfer of CTLs. Saline suspensions of CTLs labeled with DiR were constructed at a concentration of $1 \times 10^{8}$ cells $/ \mathrm{ml}$ and each tumor-bearing mouse received an infusion of $0.1 \mathrm{ml}\left(1 \times 10^{7}\right)$ labeled cells.

Fluorescence live imaging (FLI). After infusion of CTLs, each mouse was anesthetized with 2\% isoflurane (Yuyan Instruments, Shanghai, China) and FLI was performed using the Xenogen IVIS-Spectrum Imaging System (Xenogen; Caliper Life Sciences, Inc., Hopkinton, MA, USA). Imaging examination times were set as follows: Day 0, $1 \mathrm{~h}$ after infusion; 2, 4, 8 and $24 \mathrm{~h}$ after infusion (day 1); and 2, 6, 9 and 12 days after infusion. Living Image v.4.1 software (PerkinElmer, Waltham, MA, USA) was used to draw and calculate the region of interest.

Flow cytometry to detect the content of CTLs in tumor tissue. Recipient tumor-bearing mice were sacrificed $8 \mathrm{~h}$ after

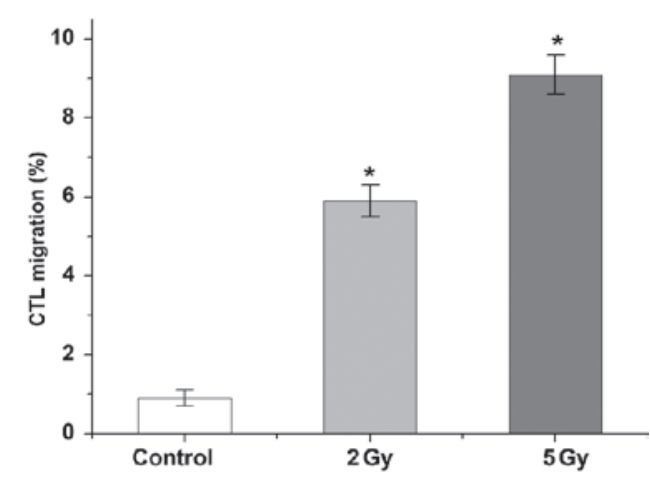

Figure 3. Assessment of migration of immunocytes mediated by LDI. Migration of CTLs towards irradiated AGS-EBV cells was increased by $\sim 6$ and 9-fold after 2 and 5 Gy LDI, respectively, compared with non-irradiated control tumor cells. Data are presented as the mean \pm standard deviation. "P $<0.05$ vs. Control. LDI, low dose irradiation; CTLs, cytotoxic T lymphocytes; AGS-EBV, gastric carcinoma cell line.

injection of DiR-labeled CTLs. The tumors of these mice were isolated. Single cell suspensions of tumor tissues were prepared and the CTL content was detected using flow cytometry. The single cell suspension was adjusted to $1-2 \times 10^{7}$ cells $/ \mathrm{ml}$ and $1 \mathrm{ml}$ was inserted into a $1.5-\mathrm{ml}$ Eppendorf tube. Following washing two times with PBS, the cells were suspended in $5 \mu \mathrm{l}$ CD3 antibodies (BD Biosciences; cat. no. 6203-25, 1:20 dilution) respectively, and incubated in the dark at $4^{\circ} \mathrm{C}$ for $30 \mathrm{~min}$. Subsequently, cells were washed with PBS two times again and suspended to $1 \mathrm{ml}$ PBS prior to being examined using a flow cytometer. Analysis was performed using CellQuest software (Version 5.1, BD Biosciences).

Statistical analysis. All statistical analyses were performed using SPSS v.17.0 software (SPSS, Inc., Chicago, IL, USA). One way analysis of variance and Newman-Keuls multiple comparison tests were used to compare the differences between multiple groups. Unpaired two-tailed Student's t-tests 


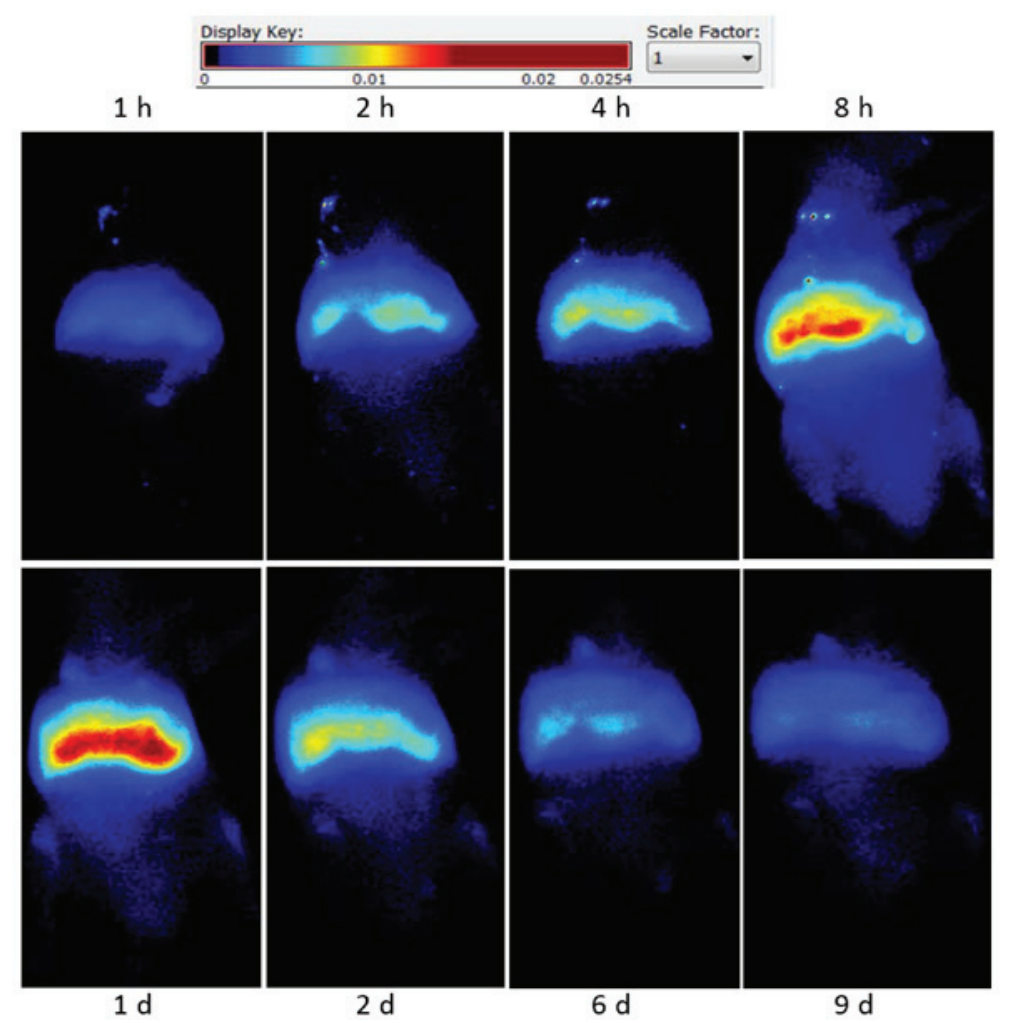

Figure 4. Fluorescence live imaging of accumulation of CTLs in tumor sites following local LDI. Intravenously-infused CTLs displayed enhanced accumulation in tumor sites after $2 \mathrm{~Gy} \mathrm{LDI}$, with levels peaking $8 \mathrm{~h}$ after LDI, compared with the non-LDI-treated control group. Enhanced accumulation of CTLs was not detected at un-irradiated tumor sites. CTLs, cytotoxic T lymphocytes; LDI, low dose irradiation.

were used to analyze differences between two groups. Data were presented as the mean \pm standard deviation. $\mathrm{P}<0.05$ was considered to indicate a statistically significant difference.

\section{Results}

Content of LMP2A-specific CTLs. The content of LMP2A-specific T cells in the CTL population was detected by EBV-LMP2A-356 tetramer technology. The EBV-LMP2A-356 antigen peptide-loaded DC induced peripheral blood mononuclear cells into CTLs in vitro. At 14 days after the cells were cultured, a small amount $\left(5 \times 10^{6}\right)$ of CTLs were incubated with fluorescein-labelled EBV-LMP2A-356 tetramers for $30 \mathrm{~min}$, and CD3 and CD8 were subsequently labelled with a flow cytometry antibody. It was demonstrated that the EBV-LMP2A-356-specific CTLs accounted for $47.1 \%$ of $\mathrm{CD}^{+} \mathrm{T}$ cells (Fig. 1).

Radiation induces upregulation of CXCL9 and CXCL1O release in tumor cells. Chemotaxis assays were performed to detect the secretion of chemokines CXCL9 and CXCL10 by AGS-EBV cells that were able to recruit antitumor effector $\mathrm{T}$ cells. ELISA test results demonstrated that there was not significant CXCL9 and CXCL10 release in AGS-EBV cells at the basal condition (CXCL9, 30.23 $\pm 4.31 \mathrm{pg} / \mathrm{ml}$; CXCL10, 28.21 $\pm 5.34 \mathrm{pg} / \mathrm{ml})$. However, in 2 Gy-irradiated tumor cells, soluble CXCL9 and CXCL10 significantly increased by 7 -fold (CXCL9, 201.32 $\pm 21.37 \mathrm{pg} / \mathrm{ml}$; CXCL10, $218.54 \pm 18.37 \mathrm{pg} / \mathrm{ml}$ ) compared with non-irradiated tumor cells $(\mathrm{P}<0.05)$. In 5 Gy-irradiated tumor cells, soluble CXCL9 and CXCL10 was significantly increased by $\sim 10$-fold (CXCL9,
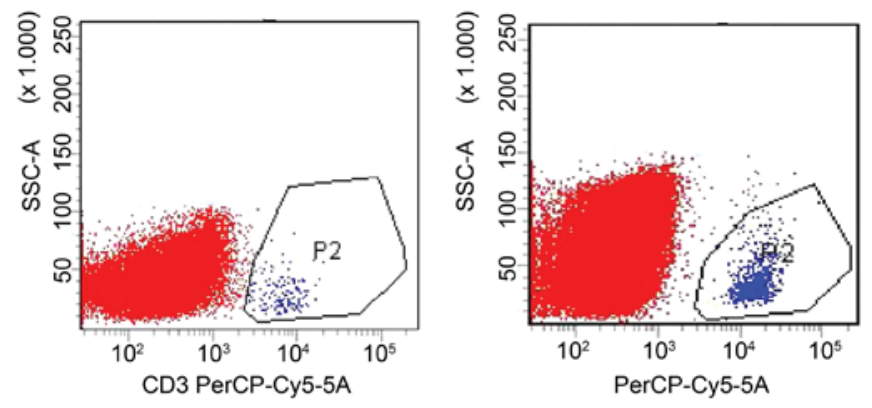

Figure 5. Effect of LDI on CTL migration to tumor sites determined by flow cytometry. In the CTL-i.v. group (left panel), the ratio of T lymphocytes in the single cell suspension of tumor tissues was $0.15 \pm 0.03 \%$, whereas in the CTL-i.v. + LDI group (right panel), the ratio of T lymphocytes increased by $\sim 9$-fold to $1.45 \pm 0.14 \%(\mathrm{P}<0.05)$. CTLs, cytotoxic T lymphocytes; LDI, low dose irradiation; CTL-i.v., intravenous injection of cytotoxic T lymphocytes; CTL-i.v.+LDI, intravenous injection of cytotoxic T lymphocytes plus low dose irradiation.

$320.47 \pm 31.47 \mathrm{pg} / \mathrm{ml}$; CXCL10, 332.56 $\pm 18.37 \mathrm{pg} / \mathrm{ml}$ ) compared with non-irradiated tumor cells $(\mathrm{P}<0.05$; Fig. 2$)$.

LDI attracts CTLs to tumor cells in vitro. Transwell migration assays demonstrated that both 2 and 5 Gy LDI promoted immune cell migration to tumor sites. There was no evident immune cell migration towards non-irradiated gastric cancer cells $(0.9 \pm 0.2 \%)$. As expected, migration of CTLs towards irradiated AGS-EBV cells was significantly increased $\sim 6$-fold after 2 Gy LDI $(5.9 \pm 0.4 \%)$ and $\sim 9$-fold after 5 Gy LDI (9.1 $\pm 0.5 \%$; $\mathrm{P}<0.05$; Fig. 3). 


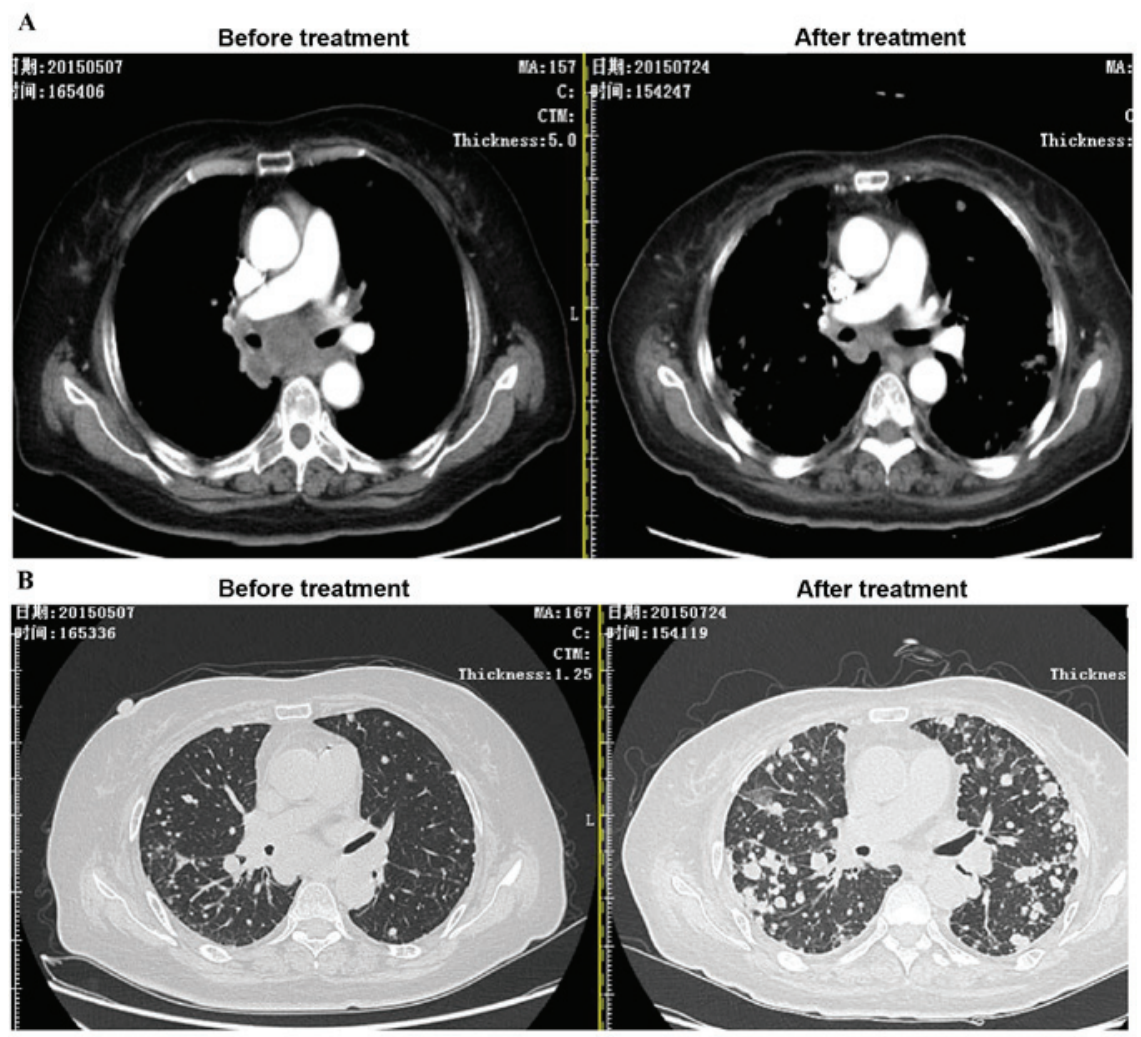

Figure 6. Effect of LDI on CTL migration to tumor site in a cancer patient with a stage IV metastatic mediastinum tumor. Following two courses of LDI + adoptive cellular immunotherapy treatment, (A) tumor size was markedly reduced; however, (B) the lesions induced by the radiotherapy field increased. LDI, low dose irradiation; CTL, cytotoxic T lymphocyte.

Distribution of CTLs in tumor-bearing mice. DiR-labeled CTLs were injected into tumor-bearing nude mice, intravenously. Fluorescent signal intensity was observed at different times using fluorescence imaging. CTLs were predominantly accumulated in the liver and spleen region, their levels peaked at $24 \mathrm{~h}$ and gradually decreased and cleared at 9 days (Fig. 4).

Effect of LDI on the distribution of CTLs in tumor-bearing mice. Intravenous-infused CTLs displayed enhanced accumulation in tumor sites after 2 Gy LDI, with CTL levels peaking at $8 \mathrm{~h}$. These levels then gradually decreased and cleared at $\sim 24 \mathrm{~h}$ (Fig. 4). Enhanced accumulation of CTLs at un-irradiated tumor sites was not observed in the control group. Living Image v.4.1 software was used to draw and calculate the region of interest.

Effect of LDI on the migration of CTLs to tumor sites in vivo. Flow cytometry results demonstrated that only a small proportion of lymphocytes were detected in the tumor tissue of the CTL-i.v. group, with a ratio of $\mathrm{T}$ lymphocytes in the single cell suspension of tumor tissue of $0.15 \pm 0.03 \%$. A significantly increased number of lymphocytes ( $\sim 9$-fold) was detected in the CTL-i.v. + LDI group compared with the CTL-i.v. group, with a ratio of $\mathrm{T}$ lymphocytes in the single cell suspension of tumor tissue of $1.45 \pm 0.14 \%(\mathrm{P}<0.05$; Fig. 5).

Effect of LDI in a patient with metastatic gastric cancer. In a case study, ACI combined with 2 Gy LDI was performed on a stage IV cancer patient with a metastatic mediastinum tumor. As expected, after two courses of LDI + ACI treatment, the size of the mediastinum tumor was markedly reduced compared to the tumor size before treatment; however, the lesions as a result of radiotherapy were increased (Fig. 6).

\section{Discussion}

In the present study it was demonstrated, for the first time, that 2 Gy LDI is able to markedly upregulate the release of chemokines CXCL9 and CXCL10 from human gastric cancer cells. It was also demonstrated that local tumor LDI increases the migration of activated CTLs to tumor sites, both in vitro and in vivo.

An essential requirement for cancer immunotherapy is activation of antigen-specific $\mathrm{T}$ cells and their homing to tumors. Although clinical studies on antitumoral immunization have reported the presence of immune effector cells in the periphery or at the injection site, the therapeutic antitumor responses remain limited $(2,15)$. The most obvious reason for the lack of clinical response is insufficient tumor infiltration of immune effector cells (4).

Radiotherapy is a standard treatment used in oncology and may be very precisely targeted to tumors to minimize immunosuppressive side effects, which is an advantage over systemic chemotherapy (16). Recently, the possibility of combining local radiotherapy with immunotherapy has been explored (17). The ability of irradiation to convert tumors into inflamed peripheral tissues may be exploited to overcome obstacles at the effector phase of the antitumor immune response and improve the therapeutic efficacy of immunotherapy (17). However, irradiation alone has been demonstrated to increase the number of 
intratumoral myeloid-derived suppressor cells (MDSCs) (18). The number of MDSCs has not been demonstrated to increase when irradiation is combined with immunization (19). Furthermore, Klug et al (5) demonstrated that LDI of 2 Gy resulted in the highest ratio of effector $\mathrm{T}$ cells to $\mathrm{FoxP}^{+}$immunosuppressive regulatory $\mathrm{T}$ cells when combined with adoptive $\mathrm{T}$ cell transfer. The ratio of antigen-specific $\mathrm{CD} 8^{+} \mathrm{T}$ cells and MDSCs in tumors increased by $~ 85$-fold compared with the control group (5). Based on these findings, the present study used 2 Gy LDI. Additionally, LDI induces minimal damage to surrounding normal tissue, can be widely applied and is particularly suitable for recurrent tumors that have received radiotherapy previously (20). LDI may be more suitable as a clinical treatment when combined with immunotherapy.

Effective $\mathrm{T}$ cell migration into peripheral tissues occurs at sites of infection-induced inflammation, through interaction with endothelial cells and is guided by chemokines secreted by activated cells $(21,22)$. A study by Matsumura et al (23) demonstrated that ionizing radiation markedly enhanced the secretion of CXCL16 by murine and human breast cancer cells, resulting in the recruitment of antitumor effector T cells to sites of inflammation. Soluble CXCL9 and CXCL10 has been demonstrated to induce strong chemotaxis of activated $\mathrm{T}$ cells and natural killer cells, which express high levels of $\mathrm{CXCR}^{+}(2,24,25)$. Similarly, the results of the present study indicate that LDI-treated EBV-AGS tumor cells induce strong CXCL9/CXCL10-dependent chemotaxis of activated CD8 ${ }^{+}$ cells in vitro (Fig. 3 ), and that local tumor LDI in vivo may enhance the recruitment of tumor-specific $\mathrm{CD} 8^{+}$cells (Fig. 4).

The mechanisms by which LDI regulates trafficking of $\mathrm{T}$ cells to solid tumors remain largely undefined. Klug et al (5) also demonstrated that local neoadjuvant LDI resulted in normalization of aberrant vasculature and efficient recruitment of tumor-specific T cells in human pancreatic carcinomas, which led to $\mathrm{T}$ cell-mediated tumor rejection and prolonged survival in xenotransplant murine tumor models. From the present study, it is evident that 2 Gy LDI is able to markedly enhance the secretion of chemokines CXCL9 and CXCL10 by human gastric cancer cells, which have an important role in the recruitment of tumor-specific CTLs to tumor sites. Localized 2 Gy LDI induces enhanced intratumoral levels of immune cell populations that are crucial for stimulating the antitumor responses in the effector phase of the antitumor immune response. The underlying molecular mechanisms are, at least partly, due to irradiation-induced changes in chemokine levels.

In conclusion, the present study demonstrates that local $2 \mathrm{~Gy}$ LDI of tumors induces upregulation of chemokine secretion, resulting in the recruitment of activated $\mathrm{T}$ cells to the tumor. These results suggest that LDI may be an easily translatable strategy to overcome immune barriers at the effector phase of ACI.

\section{References}

1. Smyth MJ, Ngiow SF, Ribas A and Teng MW: Combination cancer immunotherapies tailored to the tumour microenvironment. Nat Rev Clin Oncol 13: 143-158, 2016.

2. Abastado JP: The next challenge in cancer immunotherapy: Controlling T-cell traffic to the tumor. Cancer Res 72: 2159-2161, 2012.

3. Fridman WH, Galon J, Pagès F, Tartour E, Sautès-Fridman C and Kroemer G: Prognostic and predictive impact of intra- and peritumoral immune infiltrates. Cancer Res 71: 5601-5605, 2011.
4. Draghiciu O, Nijman HW and Daemen T: From tumor immunosuppression to eradication: Targeting homing and activity of immune effector cells to tumors. Clin Dev Immunol 2011: 439053, 2011.

5. Klug F, Prakash H, Huber PE, Seibel T, Bender N, Halama N, Pfirschke C, Voss RH, Timke C, Umansky L, et al: Low-dose irradiation programs macrophage differentiation to an $\mathrm{iNOS}^{+} / \mathrm{M} 1$ phenotype that orchestrates effective $\mathrm{T}$ cell immunotherapy. Cancer Cell 24: 589-602, 2013.

6. Domanska UM, Kruizinga RC, Nagengast WB, Timmer-Bosscha H, Huls G, de Vries EG and Walenkamp AM: A review on CXCR4/CXCL12 axis in oncology: No place to hide. Eur J Cancer 49: 219-230, 2013.

7. Kang TH, Mao CP, Lee SY, Chen A, Lee JH, Kim TW, Alvarez RD, Roden RB, Pardoll D, Hung CF and Wu TC: Chemotherapy acts as an adjuvant to convert the tumor microenvironment into a highly permissive state for vaccination-induced antitumor immunity. Cancer Res 73: 2493-2504, 2013.

8. Marchesi F, Grizzi F, Laghi L, Mantovani A and Allavena P: Molecular mechanisms of pancreatic cancer dissemination: The role of the chemokine system. Curr Pharm Des 18: 2432-2438, 2012.

9. Franciszkiewicz K, Boissonnas A, Boutet $\mathrm{M}$, Combadière C and Mami-Chouaib F: Role of chemokines and chemokine receptors in shaping the effector phase of the antitumor immune response. Cancer Res 72: 6325-6332, 2012.

10. Ohtani H, Jin Z, Takegawa S, Nakayama T and Yoshie O: Abundant expression of CXCL9 (MIG) by stromal cells that include dendritic cells and accumulation of CXCR3 $3+\mathrm{T}$ cells in lymphocyte-rich gastric carcinoma. J Pathol 217: 21-31, 2009.

11. Frangioni JV: In vivo near-infrared fluorescence imaging. Curr Opin Chem Biol 7: 626-634, 2003.

12. He X, Gao J, Gambhir SS and Cheng Z: Near-infrared fluorescent nanoprobes for cancer molecular imaging: Status and challenges. Trends Mol Med 16: 574-583, 2010.

13. DU X, Wang X, Ning N, Xia S, Liu J, Liang W, Sun H and $\mathrm{Xu}$ Y: Dynamic tracing of immune cells in an orthotopic gastric carcinoma mouse model using near-infrared fluorescence live imaging. Exp Ther Med 4: 221-225, 2012.

14. Liu G, Yao K, Wang B, Chen Y, Zhou F, Guo Y, Xu J and Shi H: Immunotherapy of Epstein-Barr virus associated malignancies using mycobacterial HSP70 and LMP2A356-364 epitope fusion protein. Cell Mol Immunol 6: 423-431, 2009.

15. Draghiciu O, Nijman HW and Daemen T: From tumor immunosuppression to eradication: Targeting homing and activity of immune effector cells to tumors. Clin Dev Immunol 2011: 439053, 2011

16. Demaria S, Pilones KA, Vanpouille-Box C, Golden EB and Formenti SC: The optimal partnership of radiation and immunotherapy: From preclinical studies to clinical translation. Radiat Res 182: 170-181, 2014.

17. Reynders K and De Ruysscher D: Radiotherapy and immunotherapy: Improving cancer treatment through synergy. Prog Tumor Res 42: 67-78, 2015.

18. Kalbasi A, June $\mathrm{CH}$, Haas $\mathrm{N}$ and Vapiwala N: Radiation and immunotherapy: A synergistic combination. J Clin Invest 123: 2756-2763, 2013

19. Soukup K and Wang X: Radiation meets immunotherapy - a perfect match in the era of combination therapy? Int J Radiat Biol 91: 299-305, 2015.

20. Patel NR, Lanciano R, Sura K, Yang J, Lamond J, Feng J, Good M, Gracely EJ, Komarnicky L and Brady L: Stereotactic body radiotherapy for re-irradiation of lung cancer recurrence with lower biological effective doses. J Radiat Oncol 4: 65-70, 2015.

21. Bryant J, Ahern DJ and Brennan FM: CXCR4 and vascular cell adhesion molecule 1 are key chemokine/adhesion receptors in the migration of cytokine-activated T cells. Arthritis Rheum 64: 2137-2146, 2012.

22. Lim JY, Gerber SA, Murphy SP and Lord EM: Type I interferons induced by radiation therapy mediate recruitment and effector function of CD8(+) T cells. Cancer Immunol Immunother 63: 259-271, 2014.

23. Matsumura S, Wang B, Kawashima N, Braunstein S, Badura M, Cameron TO, Babb JS, Schneider RJ, Formenti SC, Dustin ML and Demaria S: Radiation-induced CXCL16 release by breast cancer cells attracts effector T cells. J Immunol 181: 3099-3107, 2008.

24. Zhu Q, Han X, Peng J, Qin H and Wang Y: The role of CXC chemokines and their receptors in the progression and treatment of tumors. J Mol Histol. 43: 699-713, 2012.

25. Balkwill FR: The chemokine system and cancer. J Pathol 226: 148-157, 2012. 